\title{
Effects of Exercise on Neuropathy in Streptozotocin-Induced Diabetic Rats
}

\author{
Eui Chang Lee, MD, Myeong Ok Kim, MD, PhD, Gill Ho Roh, MD, PhD, Sang Eun Hong, MD
}

Department of Physical and Rehabilitation Medicine, Inha University, Incheon, Korea

\begin{abstract}
Objective To evaluate the effects of early regular exercise and to assess the electrophysiological and histopathological findings of the rat tail nerve in relation to the timing of exercise training for swimming exercise in rats with diabetic neuropathy.

Methods We used 70 Sprague-Dawley male rats, and the experimental group comprised 60 rats, and the control group comprised 10 rats. Diabetes was induced by intraperitoneal injection of streptozotocin. Blood glucose concentrations were measured in tail vein blood samples. The experimental group was divided into 6 subgroups according to insulin treatment and swimming exercise: group 1, diabetic control; group 2, insulin treated; group 3 , insulin untreated with early swimming exercise; group 4, insulin treated and early swimming exercise; group 5, insulin treated and late swimming exercise; and group 6, insulin untreated with late swimming exercise. Sensory and motor nerve conduction studies were performed weekly up to the 13th week using rat tail nerves. The effect on structural diabetic neuropathy was assessed by morphometry and ultrastructural examination of the rat tail nerve fiber at the 14 th week.

Results An exercise effect was observed in the insulin treated groups, but it was not observed in the insulin untreated groups. The sensory nerve conduction study in the rat tail revealed significantly prolonged latency and decreased amplitude in groups 1 and 6, and a further delay was observed in group 5 when compared to group 4 . Decreased thickness of myelin was found in groups 1 and 6 through morphometry.

Conclusion Early regular exercise programs in addition to conventional insulin treatment may retard the progression of diabetic peripheral neuropathy.
\end{abstract}

Keywords Nerve tissue, Streptozotocin, Diabetic neuropathies, Swimming, Neural conduction

\section{INTRODUCTION}

Diabetic peripheral neuropathy is an important com- plication of diabetes mellitus. The prevalence of diabetic neuropathy varies within the research, but approximately $60 \%$ of diabetic patients demonstrate clinical manifesta-

Received May 30, 2016; Accepted September 12, 2016

Corresponding author: Myeong Ok Kim

Department of Physical and Rehabilitation Medicine, Inha University, 27 Inhang-ro, Jung-gu, Incheon 22332, Korea. Tel: +82-32-890-2480, Fax: +82-32890-2486, E-mail:rmkmo@inha.ac.kr

ORCID: Eui Chang Lee (http://orcid.org/0000-0002-7383-0713); Myeong Ok Kim (http://orcid.org/0000-0002-5584-7714); Gill Ho Roh (http://orcid. org/0000-0002-2927-3833); Sang Eun Hong (http://orcid.org/0000-0002-9433-6642).

(c) This is an open-access article distributed under the terms of the Creative Commons Attribution Non-Commercial License (http://creativecommons.org/ licenses/by-nc/4.0) which permits unrestricted noncommercial use, distribution, and reproduction in any medium, provided the original work is properly cited. Copyright (c) 2017 by Korean Academy of Rehabilitation Medicine 
tions of peripheral neuropathy [1-3]. Persistent hyperglycemia, metabolic and endocrinal changes, inflammatory effects of obesity and dyslipidemia appear to play an important role in the development of diabetic neuropathy [4]. When it comes to diabetic neuropathy, a diabetic condition promotes delay in nerve regeneration [5].

Aerobic exercise has been shown to improve the symptoms of neuropathy and accelerate re-growth and prevent or revert morphometric alterations in the injured nerve, especially those in small diameter fibers $[4,5]$. However, opposing opinions on the effectiveness of exercise have also been reported. Van Meeteren et al. [6] have reported that exercises like treadmill or swimming can cause stressful conditions in experimental animals, and consequently can have negative effects on regeneration after nerve injury.

Electrodiagnostic studies are the most objective and quantitative measures of nerve function, and electrodiagnostic changes are even detected early in the course of diabetic neuropathy. Thus, electrodiagnostic studies are used as a valuable tool in large clinical studies. With respect to symptomatic diabetic neuropathy, a study reported that slowing of nerve conduction velocity owing to demyelination is the main feature of the condition [7]. Also, a decrease in the amplitude is considered to be more important [8]. However, controversy remains as to which factors affect the disease first. Some researchers state that demyelination is the primary lesion in the early stage of the disease $[7,9]$ but other researchers state that the disease is associated with a progressive distal axonopathy [10]. Demyelination and axonal degeneration are often considered to co-exist $[7,11]$.

The protective and therapeutic effects of exercise training on diabetic neuropathy have been established in many previous studies. However, very few data on the timing of exercise training were reviewed. In this study, we wanted to evaluate the differences in effects of swimming exercise on nerves according to the timing of exercise training in streptozotocin (STZ)-induced diabetic rats. Also, we wanted to investigate the electrophysiological and histopathological findings in which pathologic changes, axonal or demyelinating lesions, are predominant in relation to the duration of exercise in rats with diabetic neuropathy.

\section{MATERIALS AND METHODS}

\section{The experimental animal group}

We used 8-week-old, healthy, young adolescent Sprague-Dawley male rats $(\mathrm{n}=80$; Orient Bio Inc., Seongnam, Korea) which weighed 170-200 g. They were raised (5 rats in each cage) at homeostatic temperatures and humidity, and were maintained on the same diet with sufficient water.

Blood glucose concentrations in all rats were measured in tail vein blood samples using Accutrend Alpha (Roche Diagnostics Inc., Somerville, MA, USA) before induction of anesthesia.

\section{Induction of diabetes}

Eighty rats were divided into the following two groups: the experimental group comprised 70 rats and the normal control group comprised 10 rats. Diabetes was induced by intraperitoneal injection of a single dose of STZ ( $65 \mathrm{mg} / \mathrm{kg}$ body weight; Sigma, St Louis, MO, USA). STZ was freshly dissolved in a 0.1-M citrate buffer ( $\mathrm{pH} 4.5$ ) before injection and administered to the rats in a nonfasting condition. One week after the injection, blood glucose levels of all rats were tested. Rats with a glucose concentration exceeding $300 \mathrm{mg} / \mathrm{dL}$ were considered diabetic [12].

From the experimental group comprising 70 rats, 7 rats in whom diabetes was not induced and 3 rats in poor health were excluded. Overall, 60 rats in the experimental group were randomly divided into 6 groups according to how insulin treatment was administered and according to the swimming exercise groups (Fig. 1). The groups were as follows: group 1, diabetic group with no intervention; group 2, insulin treated group; group 3, insulin untreated with early swimming exercise; group 4 , insulin treated and early swimming exercise; group 5, insulin treated and late swimming exercise; and finally, group 6, insulin untreated with late swimming exercise (Table 1).

These 60 rats with diabetes were lightly injected with $2 \mathrm{U}$ of NPH-insulin (Insulatard) subcutaneously in the first week to prevent a rapid increase in blood glucose levels. Intermediate-acting insulin and NPH-insulin (Insulatard) were administered subcutaneously at fixed times, $2 \mathrm{U}$ every time before the swimming exercise program for 13 weeks in groups 2, 4, and 5 . 


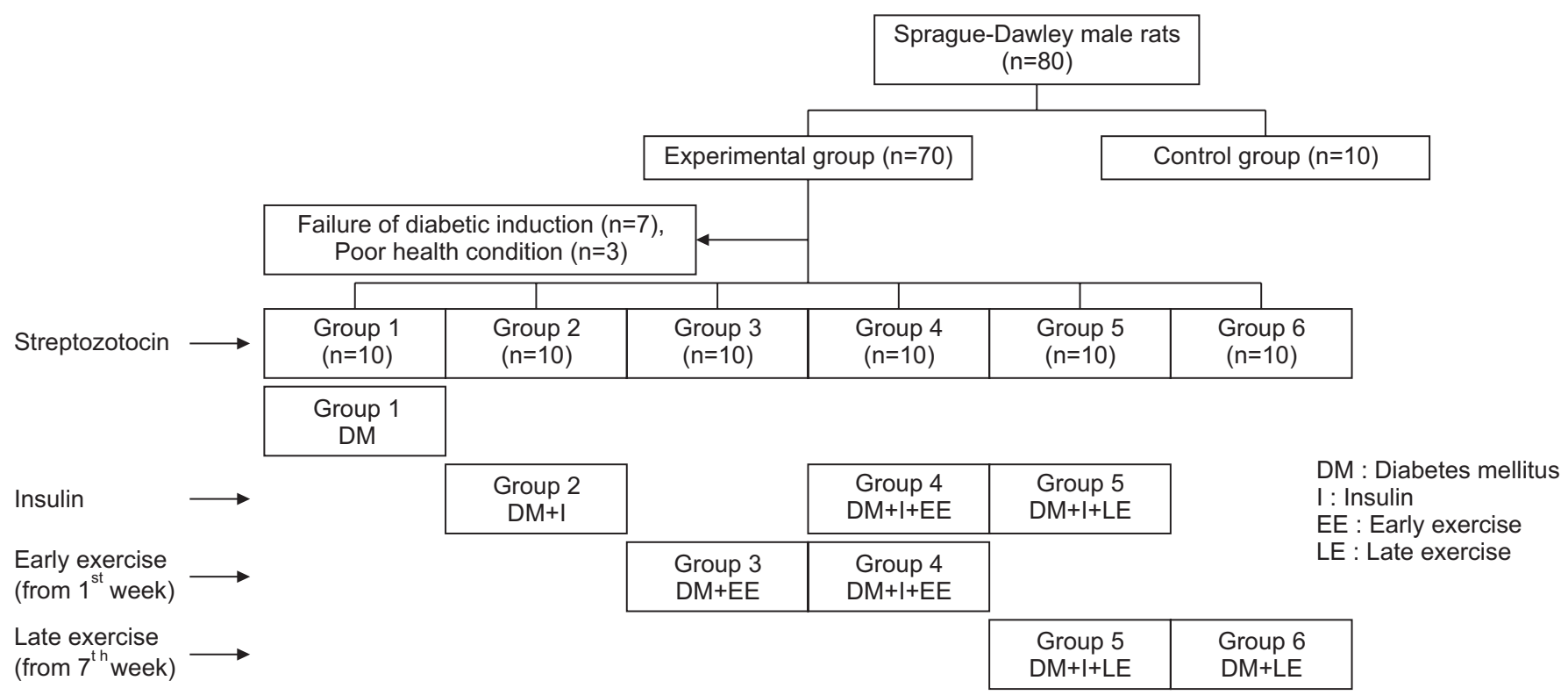

Fig. 1. Flow chart of the experiment.

Table 1. Classification of the experimental groups

\begin{tabular}{lcccc}
\hline & $\begin{array}{c}\text { Adminis- } \\
\text { tration of } \\
\text { streptozotocin }\end{array}$ & $\begin{array}{c}\text { Adminis- } \\
\text { tration of } \\
\text { insulin }\end{array}$ & $\begin{array}{c}\text { Early } \\
\text { exercise }\end{array}$ & $\begin{array}{c}\text { Late } \\
\text { exercise }\end{array}$ \\
\hline Control & $\times$ & $\times$ & $\times$ & $\times$ \\
Group 1 & 0 & $\times$ & $\times$ & 0 \\
\hline Group 2 & o & 0 & $\times$ & $\times$ \\
Group 3 & o & $\times$ & 0 & $\times$ \\
\hline Group 4 & o & o & 0 & $\times$ \\
Group 5 & 0 & 0 & $\times$ & 0 \\
\hline Group 6 & 0 & $\times$ & $\times$ & 0 \\
\hline
\end{tabular}

\section{Exercise training}

Swimming was started from the 1st week in groups 3 and 4 (the early swimming exercise group). Exercise was performed 1 hour after insulin injection. Two bath tubs (55- $\mathrm{cm}$ wide, $107-\mathrm{cm}$ long, and $75-\mathrm{cm}$ in height) were used. Five rats were placed in each tub and exercised for 30 minutes each day, 5 days a week. According to the method used in the report by Pyun et al. [1], the depth of the pool was kept high enough so that their legs did not touch the ground and the rats could swim freely in the bath tubs. The temperature of the tub was kept at $32^{\circ} \mathrm{C}-$ $36^{\circ} \mathrm{C}$. Rats were not allowed to rest while swimming, and each rat was continuously monitored so that it did not harass the other rats. From 7 weeks of insulin injection, exercise was administered to groups 5 and 6 (the late swimming exercise group). Thus, a 13-week exercise program was performed for the early exercise group and a 7-week exercise program was performed for the late exercise group.

\section{Induction of anesthesia}

For achieving high levels of safety and to have a lesser effect on blood glucose levels, the inhalational anesthetic, enflurane (Arillein), was used. In order to rule out any anesthetic effect on the blood glucose level, blood glucose concentrations in all rats were measured before induction of anesthesia. Induction was performed in all rats every week just before performing the nerve conduction study (NCS) with $100 \%$ oxygen plus $2 \%$ of enflurane via spontaneous inhalation.

\section{Nerve conduction study}

Temperatures of the tails were measured using an electronic thermometer (Dantec, Copenhagen, Denmark), and they were kept at $32^{\circ} \mathrm{C}-36^{\circ} \mathrm{C}$ using a heater. Then, electrodiagnostic examinations for the sensory and motor nerve conduction studies were performed in rats every week using KeyPoint (Dantec, Copenhagen, Denmark).

Using the method presented in the study of Kim et al. [13], an orthodromic sensory NCS was performed. Electrical stimulations were applied at a point $8 \mathrm{~cm}$ away 
A

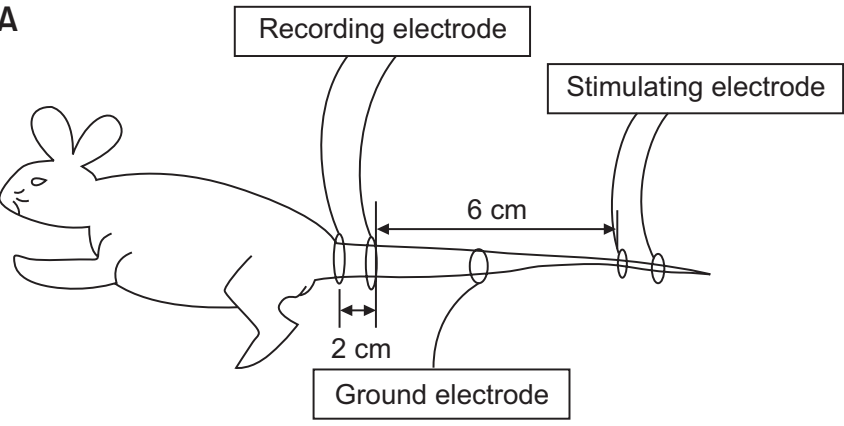

B

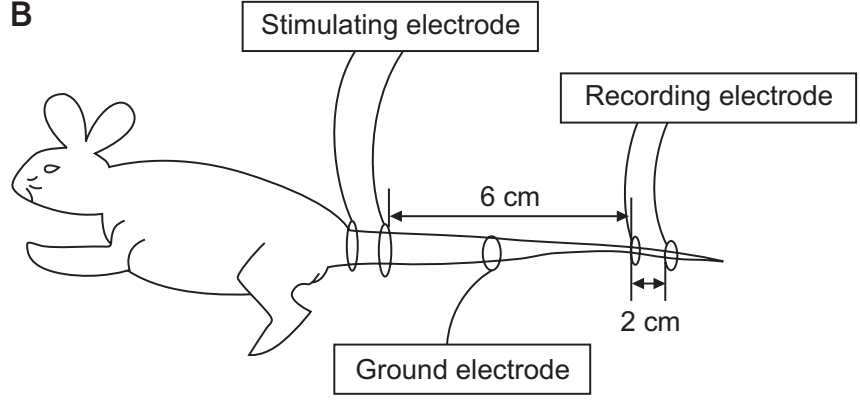

Fig. 2. (A) Measurements of the orthodromic sensory nerve conduction study. (B) Measurements of the motor nerve conduction study. from the origin of the tail using a ring electrode and a recording electrode was placed $6 \mathrm{~cm}$ away from the stimulating electrode (Fig. 2A). In the motor NCS, stimulation was applied at the origin of the tail and the recording electrode was placed $6 \mathrm{~cm}$ away from the stimulation point. The active and reference electrodes were placed $2 \mathrm{~cm}$ apart (Fig. 2B). The bandwidth, sweep speed, and sensitivity were $2-10,000 \mathrm{~Hz}, 1 \mathrm{~ms} /$ division, and $1 \mathrm{mV} /$ division, respectively, in the motor NCS, and they were $20-2,000 \mathrm{~Hz}, 1 \mathrm{~ms} /$ division, and $5 \mu \mathrm{V} /$ division, respectively, in the sensory NCS.

In the sensory NCS, the duration was set at $0.1 \mathrm{~ms}$, frequency was set at $2.0 \mathrm{~Hz}$, and 20 stimulations were applied, and then the values were averaged. Amplitude was measured at peak to peak distances (Fig. 3A). In the motor NCS, onset latency and peak to peak amplitude were measured when supramaximal stimulation intensity was used (Fig. 3B).

\section{Histopathologic findings}

At the 14th week, 5 rats from each group were randomly selected and their tails were sampled. The tails of rats were dissected for histopathologic examination to ascertain demyelination or axonal degeneration. The dissected nerves were cleaned with $4 \%$ paraformaldehyde-glutar-
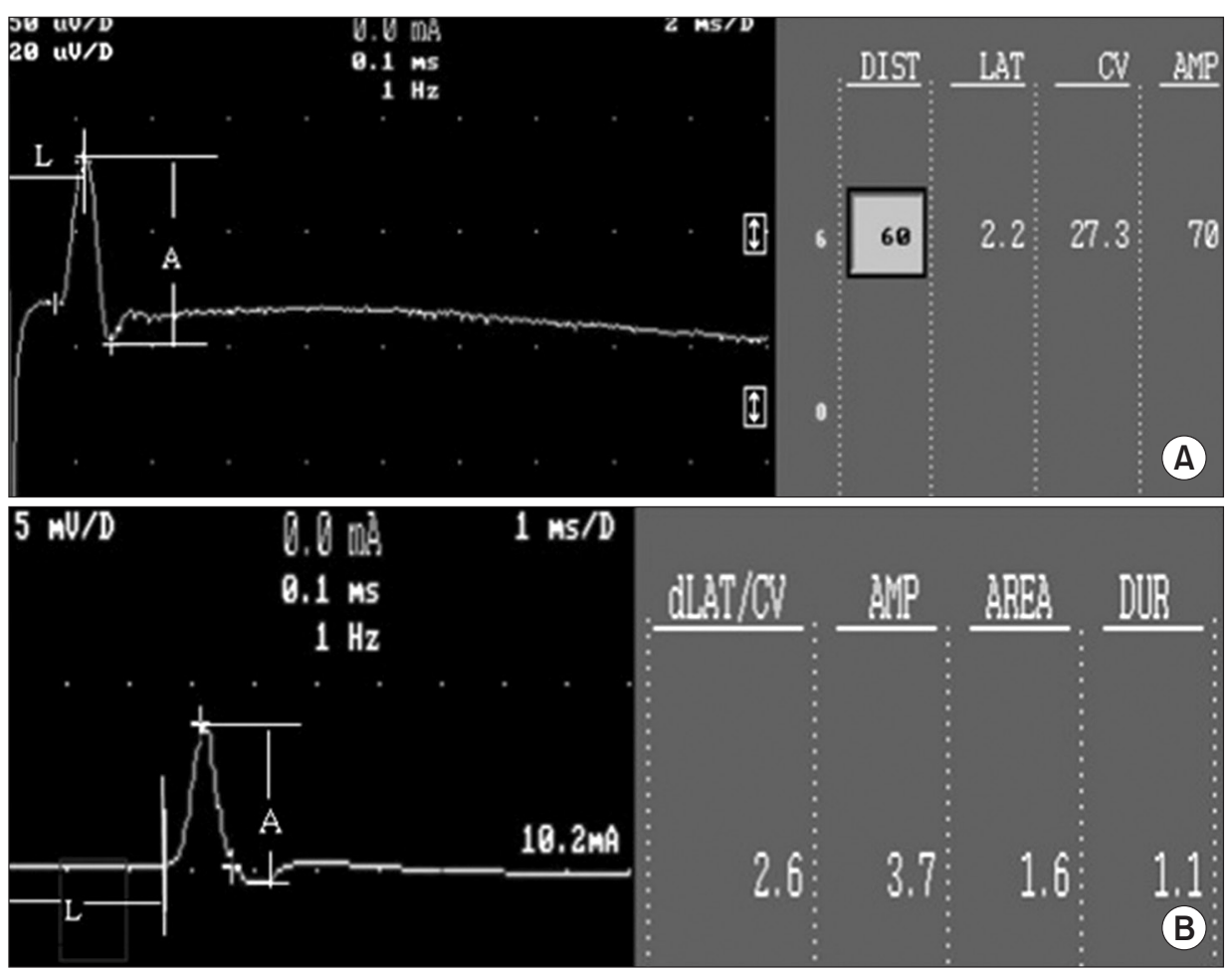

Fig. 3. (A) An example of sensory nerve action potential of the rat tail nerve. (B) An example of compound muscle action potential of the rat tail nerve. A, amplitude; L, latency. 
aldehyde $\left(4^{\circ} \mathrm{C}\right.$, phosphate buffer, $\mathrm{pH}$ 7.4) and post-fixed with $1 \% \mathrm{OsO}_{4}\left(4^{\circ} \mathrm{C}\right.$, phosphate buffer). After fixation, the specimens were washed a few times in the same buffer, and then, they were dehydrated with numerous ascending alcohol passages. Propylene oxide was added and the tail samples were embedded in Epon. Embedded sections were cut using an ultramicrotome and stained with $1 \%$ Toluidine blue, and nerve specimens were observed with a light microscope. Ultramicrotomy sections were produced, double stained using uranyl acetate and lead citrate, and were observed with a transmission electron microscope, Model H-7100 (Hitachi, Tokyo, Japan). The diameters of nerve fibers and axons were measured both longitudinally and axially, and the axes were averaged. Images were analyzed with the Imaging System KS 400 (Kontron Elektronik, Munchen, Germany).

\section{Statistical analysis}

A Mann Whitney U-test using SPSS ver. 21.0 (IBM SPSS, Armonk, NY, USA) was performed for statistical analyses. In addition, repeated measures ANOVA was used for a comparison among groups to determine the effectiveness of individual variables. Statistical significance was set at $\mathrm{p}<0.05$.

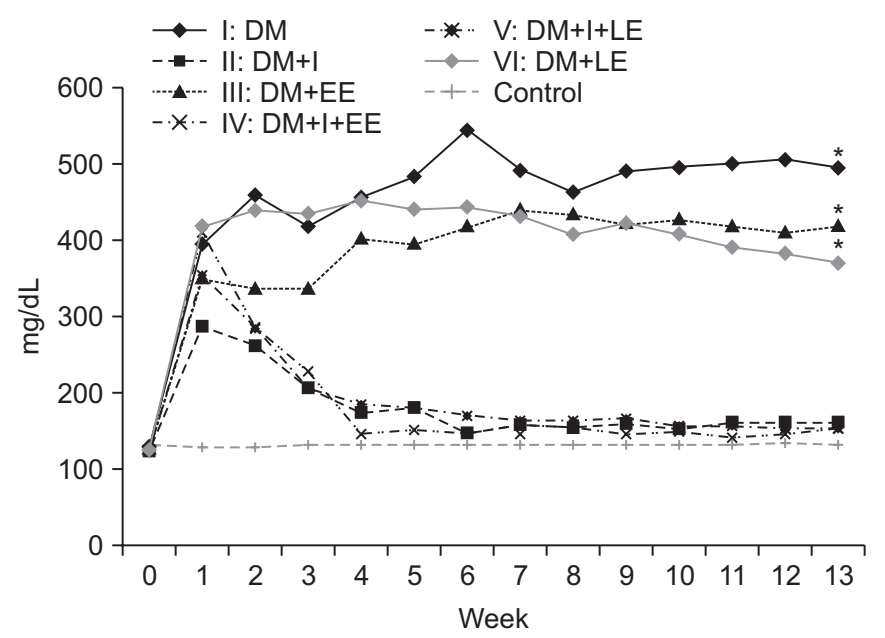

Fig. 4. Effects of insulin and exercise treatment on blood glucose levels. A significant difference was observed between insulin untreated groups and insulin treated groups from the 3 rd week $\left({ }^{*} \mathrm{p}<0.05\right.$, difference of blood glucose levels between groups 2, 4, 5 and groups $1,3,6$ ). Blood glucose level was decreased significantly in the insulin treated groups in comparison with the insulin untreated group. DM, diabetes mellitus; I, insulin treated; $\mathrm{EE}$, early exercise; LE, late exercise.

\section{RESULTS}

\section{Changes in the blood glucose level}

There was no difference in pre-insulin injection levels of blood glucose among all groups. A significant difference was observed between insulin treated groups (groups 2, 4, and 5) and insulin untreated groups (groups 1,3 , and 6 ) from the 3 rd week $(p<0.01)$. Changes in blood glucose levels with or without exercise were not significant between groups 1 and 3, and also between groups 2 and 4 ( $p>0.05$ ) (Fig. 4).

\section{Changes in body weight}

Significant differences in the reduction of body weight were observed between the insulin treated groups (groups 2, 4, and 5) and the insulin untreated groups (groups 1, 3 , and 6$)$ from the 4 th week $(\mathrm{p}<0.01)$. Changes in body weight according to exercise were not significant between groups 1 and 3, and also between groups 2 and 4 ( $p>0.05$ ) (Fig. 5).

\section{Changes in the sensory nerve conduction study}

Distal latency in the sensory NCS was delayed rapidly from the 3 rd week of diabetes induction. A significant

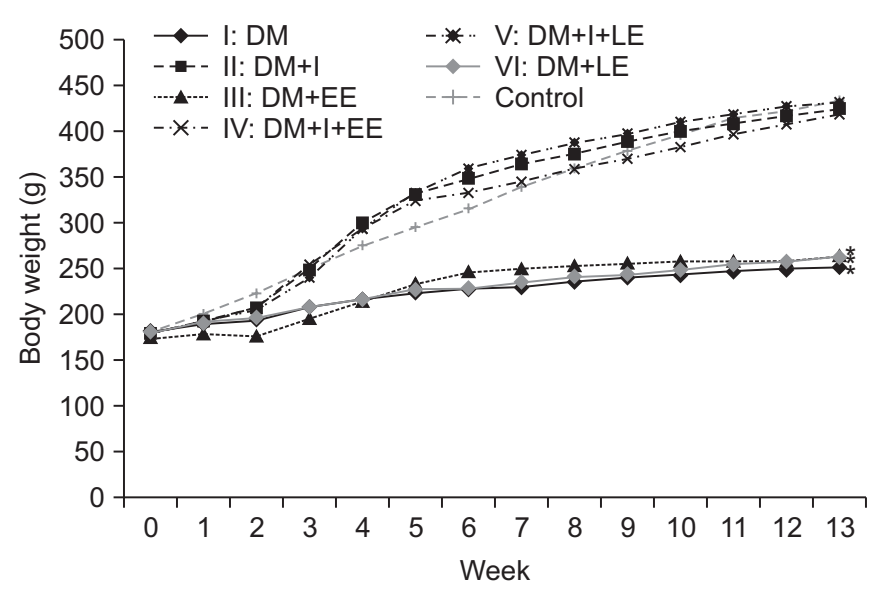

Fig. 5. Effects of insulin and exercise treatment on body weight. A significant difference was observed between the insulin untreated groups and the insulin treated groups from the 4 th week ( ${ }^{*} \mathrm{p}<0.05$, difference of body weight between groups 2, 4, 5 and groups $1,3,6$ ). Body weight was decreased significantly in the insulin untreated groups in comparison with the insulin treated group. DM, diabetes mellitus; I, insulin treated; EE, early exercise; LE, late exercise. 
difference was observed between the insulin treated groups (groups 2, 4, and 5) and the insulin untreated groups (groups 1, 3, and 6) from the 5th week. Distal latency examined in the 4 th week of swimming was less delayed in group 4 compared to the other groups. Distal latency in group 4 was less delayed compared to that in group 5 from the 7 th week. The difference in distal latency between group 2 and group 5 was not statistically significant. Differences among the control groups, groups injected with insulin, and groups without insulin were significant from the 6 th week. From the 7th week, differences among groups were maintained constantly (Fig. $6 \mathrm{~A})$.

Amplitude examined in the 5th week was temporarily highly increased in the insulin treated groups (groups 2 and 4) compared to the insulin untreated groups (groups 1 and 3), but the difference was not significant after the 5 th week. From the 5 th week, the amplitude was increased in group 4 compared to the no exercise group 2 and this difference was statistically significant. The difference in amplitude among groups 1 (no exercise), 3 (exercise from the beginning), and 6 (exercise starting from the 7 th week) was not statistically significant. From the 7th week, the amplitude was significantly increased in group 4 compared to group 5 . Amplitude in group 5 was increased from the 10th week compared to that in group 2, but, the difference was not statistically significant $(p>0.05)$ (Fig. 6B).

\section{Changes in the motor nerve conduction study}

Distal latency examined in the 3rd week was less delayed in the insulin treated groups (groups 2 and 4) compared to the insulin untreated groups (groups 1 and 3). Additionally, a significant difference was observed between the insulin untreated groups and the insulin treated groups from the 5th week. The difference was not significant among the insulin untreated groups (groups 1, 3, and 6). In addition, the difference was not significant among the insulin treated groups (groups 2, 4, and 5) (Fig. 7A).

The difference was not significant among the insulin treated groups (groups 2, 4, and 5). The difference was not significant among the insulin untreated groups (groups 1, 3, and 6). The amplitude in group 4 was increased compared to that in group 5 at the 3rd, 7th, 8th and 9th week $(\mathrm{p}<0.05)$, but from the 10th week, the difference was not significant between the groups ( $p>0.05$ ).
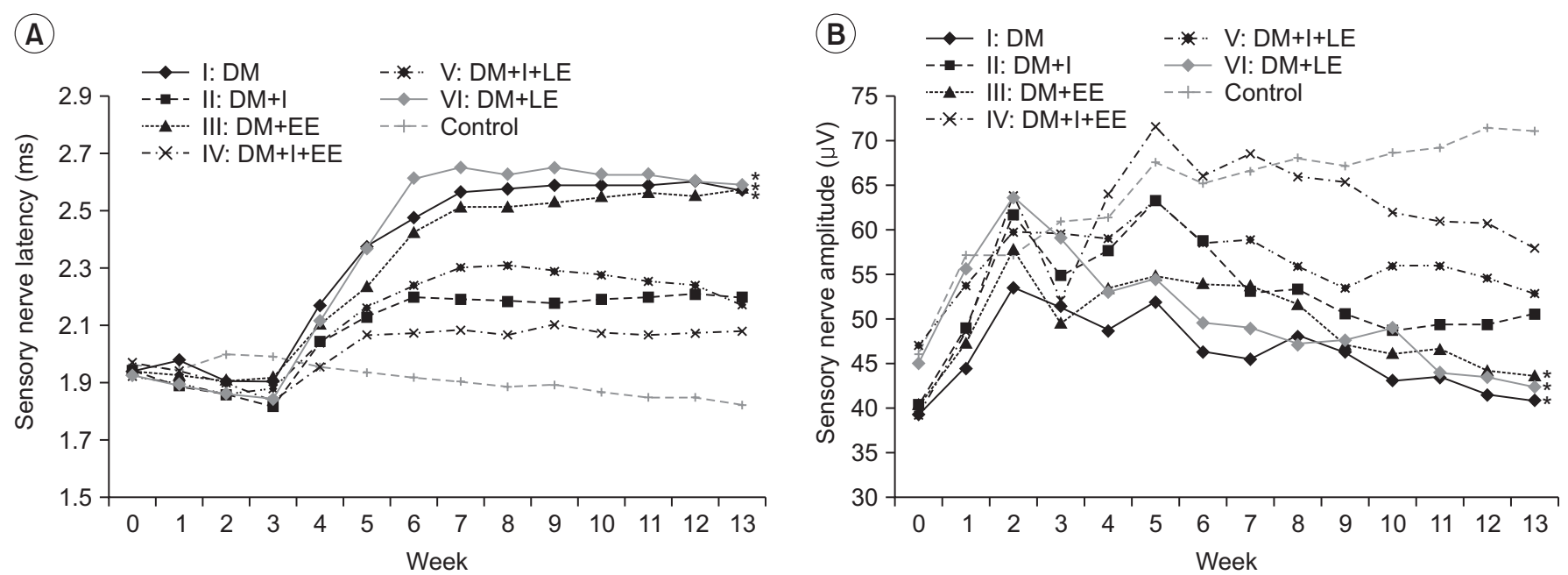

Fig. 6. (A) Effects of insulin and exercise treatment on sensory nerve latency. A significant difference was observed between the insulin untreated groups and the insulin treated groups from the 5 th week $\left({ }^{*} p<0.05\right.$, difference of distal latency in sensory nerve conduction study between groups $2,4,5$ and groups $1,3,6$ ). Sensory nerve latency was decreased significantly in the insulin treated early exercise group in comparison with the other insulin treated group from the 7th week. (B) Effects of insulin and exercise treatment on sensory nerve amplitude. A significant difference was observed between the insulin treated early exercise group and the insulin treated non-exercise group from the 5th week. Sensory nerve amplitude was increased significantly in the insulin treated early exercise group in comparison with the other insulin treated late exercise group from the 7 th week $\left({ }^{*} \mathrm{p}<0.05\right.$, difference of amplitude in sensory nerve conduction study between groups $2,4,5$ and groups $1,3,6$ ). DM, diabetes mellitus; I, insulin treated; EE, early exercise; LE, late exercise. 

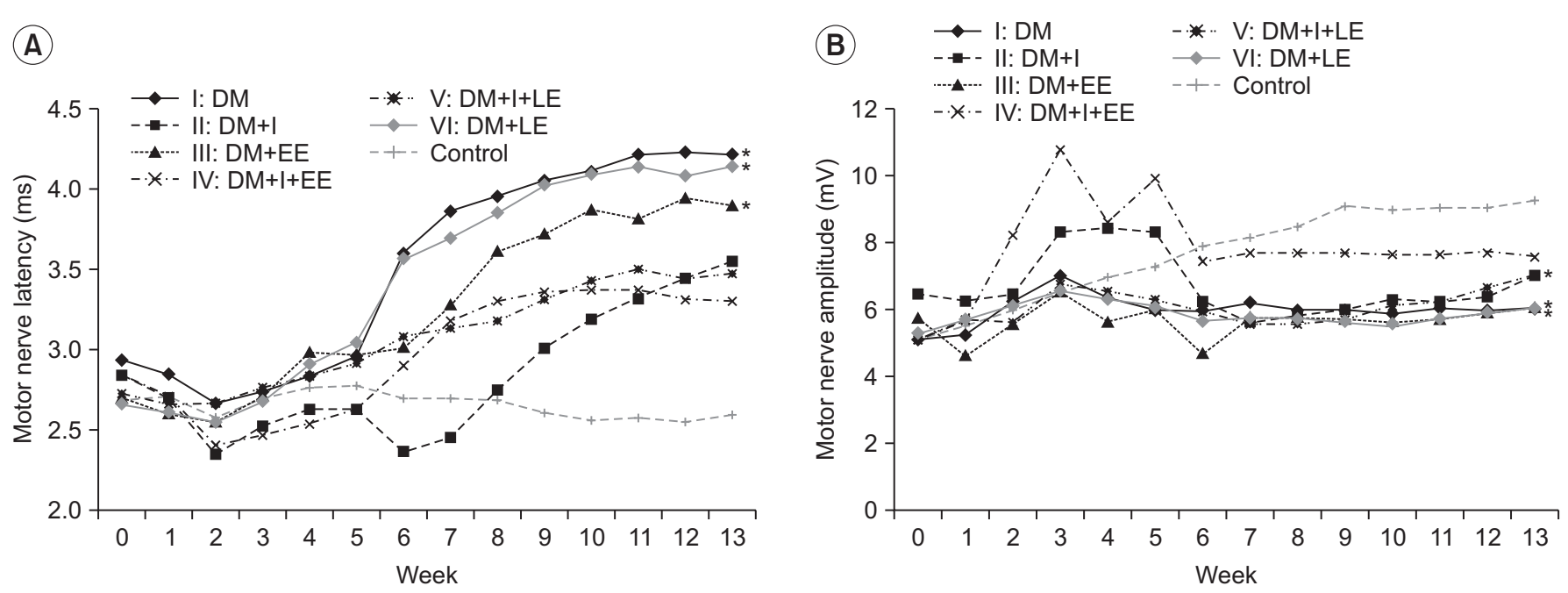

Fig. 7. (A) Effects of insulin and exercise treatment on motor nerve latency. A significant difference was observed between the insulin untreated groups and the insulin treated groups from the 5 th week $\left({ }^{*} p<0.05\right.$, difference of distal latency in motor nerve conduction study between groups 2, 4, 5 and groups $1,3,6$ ). No significantly different values were noted in the insulin untreated groups or the insulin treated groups. (B) Effects of insulin and exercise treatment on motor nerve amplitude. A significant difference was observed between the insulin treated with early exercise group and the other groups at the 3rd week and from the 7 th to the 9 th week $\left({ }^{*} \mathrm{p}<0.05\right.$, difference of amplitude in motor nerve conduction study between groups $2,4,5$ and groups $1,3,6$ ). No significant differences were noted from the values in the insulin untreated groups. DM, diabetes mellitus; I, insulin treated; EE, early exercise; LE, late exercise.

The difference was not statistically significant between groups 2 and 5 (Fig. 7B).

\section{Histopathological findings}

A light microscopic examination of rat tail nerves did not reveal any sign of demyelination or axonopathy, which are the typical findings of diabetic neuropathy (Fig. 8).

An electron microscopic examination revealed a decrease in the thickness of the myelin sheath and atrophic changes in the nerve fibers in group 1 and group 6 (Fig. 9).

The decrease in the thickness of the myelin sheath in group 1 and group 6 was statistically significant compared to the normal control group $(\mathrm{p}=0.032)$. However, decreases in the thickness of the myelin sheath in groups $2,3,4$, and 5 were not statistically significant compared to the control group ( $\mathrm{p}=0.068$ ) (Table 2).

\section{DISCUSSION}

When all results of the study were combined, groups treated with conventional insulin and/or with early exercise had a better outcome in terms of preventing the progression of diabetic neuropathy than groups treated without insulin and/or with late exercise.
A significant reduction in myelin breakdown and a remarkable improvement in degenerated axons were found after treadmill exercise (once a day for 4 consecutive weeks) [14]. Malysz et al. [5] have reported about acceleration of the hindlimb motor function recovery in diabetic rats submitted to sciatic nerve crush after 10 weeks of treadmill training. In addition, morphometric alterations in the proximal nerve portions in non-diabetic and diabetic injured rats were observed by treadmill training. There are a number of studies on treadmill exercise for diabetic neuropathy, but exercise groups divided into early and late exercise groups to determine the importance of the timing of exercise is the point of difference in this study. It can be assumed that an early exercise program provided longer time than a late exercise program to improve cardiovascular function and it had a beneficial effect on neovascularization, which in turn ameliorated impaired circulation in the nerve tissue damaged by impaired glucose metabolism. Hence, an early exercise program is considered better for retarding the progression of diabetic peripheral neuropathy than a late exercise program $[4,5]$.

It is already known that exercise ranging from $50 \%$ to $70 \%$ in terms of maximal intensity is most suitable for 30 

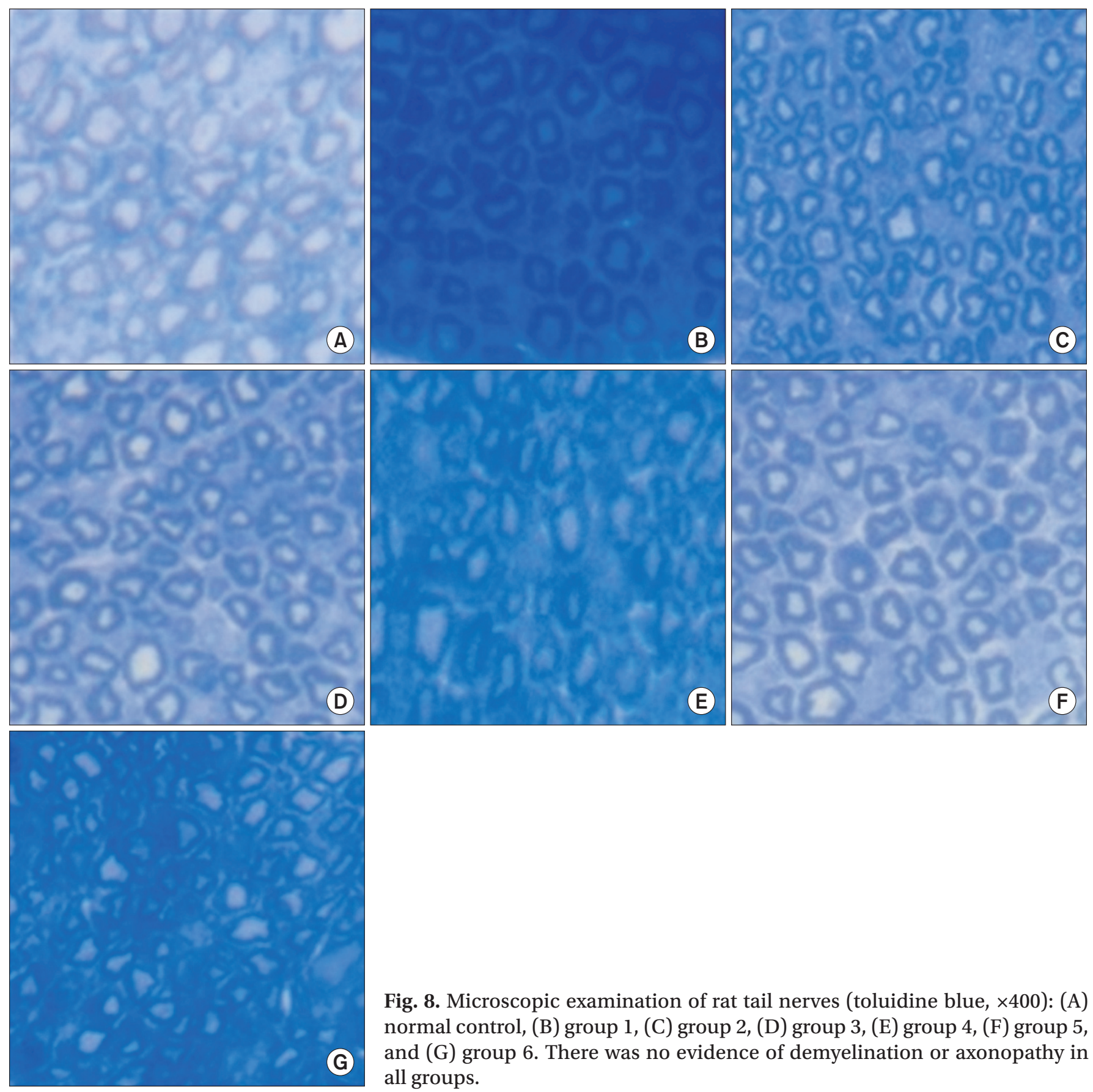

Fig. 8. Microscopic examination of rat tail nerves (toluidine blue, $\times 400$ ): (A) normal control, (B) group 1, (C) group 2, (D) group 3, (E) group 4, (F) group 5, and $(\mathrm{G})$ group 6 . There was no evidence of demyelination or axonopathy in all groups.

minutes. Kim et al. [8] allowed 70 rats with sciatic nerve injury to run on a treadmill for 4 weeks. Although the difference was not statistically significant, the diameter of muscle fiber recovered more in the 30-minute exercise group than in the 2-hour exercise group. When the duration of exercise was greatly reduced, it was difficult to achieve the targeted effects. If the duration is too long, stressful conditions can cause hyperglycemia or hypo- glycemia as well as they can possibly increase the risk of musculoskeletal injuries. In our study, when the duration of the exercise exceeded 30 minutes, rats were so tired that they either harassed other rats or died of aspiration pneumonia. Thus, the duration of exercise was limited to 30 minutes. Moreover, exercise for long durations can cause hypoglycemia during or after exercise, which can aggravate neuropathy. 

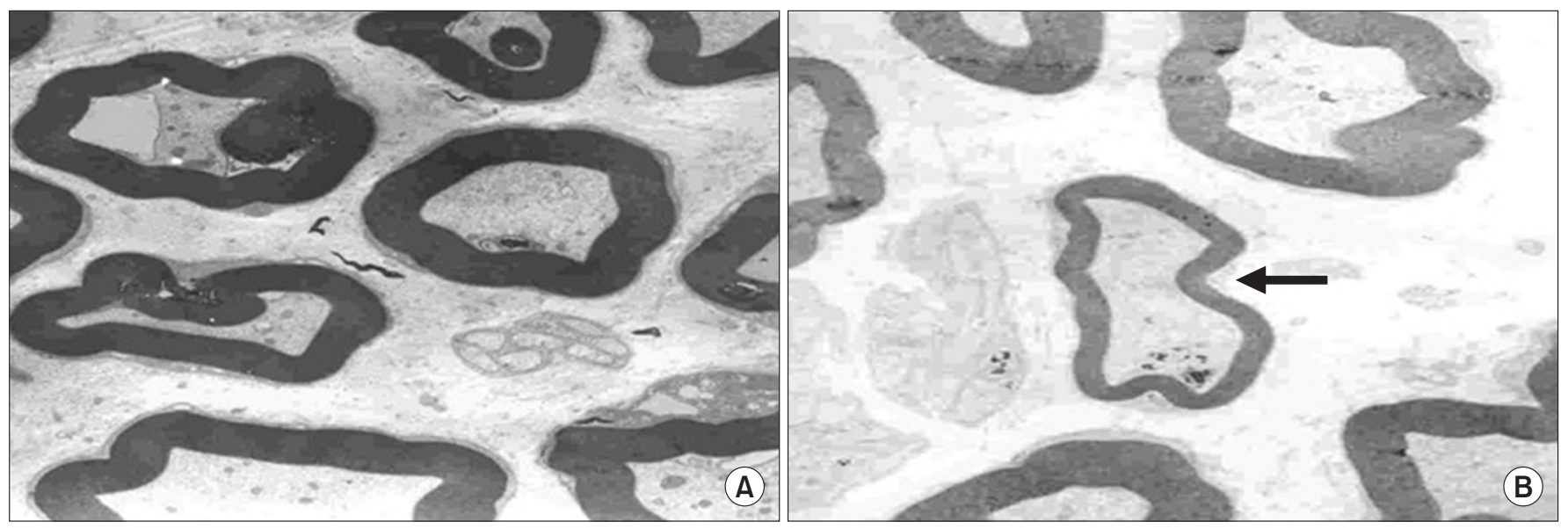

Fig. 9. Electron microscopic findings of rat tail nerves $(\times 5,000)$ : (A) control group and (B) group 1 . A disproportionately shrunk thin myelin sheath was observed (arrow).

Table 2. Rat tail nerve fiber morphology in normal and diabetic rats

\begin{tabular}{lcccc}
\hline & $\begin{array}{c}\text { Density of the myelinated } \\
\text { nerve fiber }\left(/ \mathbf{m m}^{2}\right)\end{array}$ & $\begin{array}{c}\text { Mean myelinated nerve } \\
\text { fiber diameter }(\mu \mathbf{m})\end{array}$ & $\begin{array}{c}\text { Mean axonal diameter } \\
(\mu \mathbf{m})\end{array}$ & $\begin{array}{c}\text { Myelin thickness } \\
(\mu \mathbf{m})\end{array}$ \\
\hline Control & $16,113.2 \pm 1,114.8$ & $7.60 \pm 0.43$ & $6.36 \pm 0.38$ & $0.95 \pm 0.06$ \\
Group 1 & $15,255.6 \pm 762.1$ & $7.10 \pm 0.42^{\mathrm{a})}$ & $5.94 \pm 0.49$ & $0.86 \pm 0.04^{\mathrm{a})}$ \\
\hline Group 2 & $16,912.0 \pm 1,287.1$ & $7.54 \pm 0.34$ & $6.32 \pm 0.41$ & $0.89 \pm 0.03$ \\
Group 3 & $15,569.6 \pm 1,171.4$ & $7.24 \pm 0.48$ & $5.82 \pm 0.46$ & $0.89 \pm 0.04$ \\
Group 4 & $15,556.6 \pm 1,310.8$ & $7.46 \pm 0.53$ & $6.18 \pm 0.61$ & $0.92 \pm 0.06$ \\
Group 5 & $16,222.0 \pm 1,511.2$ & $7.26 \pm 0.53$ & $6.16 \pm 0.70$ & $0.91 \pm 0.02$ \\
\hline Group 6 & $14,940.4 \pm 1,550.6$ & $7.18 \pm 0.44^{\mathrm{b})}$ & $5.70 \pm 0.48$ & $0.87 \pm 0.03^{\mathrm{b})}$ \\
\hline
\end{tabular}

Values are expressed as mean \pm standard deviation.

Control, normal control group; Group 1, diabetic control group; Group 2, insulin treated group; Group 3, insulin untreated with early swimming exercise group; Group 4, insulin treated with early swimming exercise group; Group 5, insulin treated with late swimming exercise group; Group 6, insulin untreated with late swimming exercise group.

${ }^{a)} \mathrm{p}<0.05$ between group 1 vs. control group.

${ }^{b)} \mathrm{p}<0.05$ between group 6 vs. control group.

Types of exercise depend on the individual's preference, but generally 30 minutes of intermediate intensity aerobic exercise is recommended. However, a thorough examination for any diabetes complications is needed before starting any exercise because diabetes complications can be aggravated by exercise itself. For example, high resistance exercises like heavy weight lifting are not suitable as the risk of physical trauma or microvascular complications is high. Kim and Lee [15] studied the effects of treadmill and swimming exercises on nerves in diabetic rats. Increased Schwann cell apoptosis was found in the distal peripheral nerves in groups of landbased endurance exercises. It was suggested that treadmill exercise may have a detrimental effect on peripheral nerves in rats with diabetes. Moreover, according to another study [16], treadmill training may cause foot injury during exercise, which could lead to some complications like diabetic foot. Therefore, in this study, swimming was preferred and it was chosen for proper aerobic exercise.

The mechanism of diabetic neuropathy is still controversial. In general, it is thought that metabolic disorders cause injury to neuronal cells and Schwann cells, and that microvascular occlusions may provoke nerve ischemia [17-19]. Kwon et al. [7] reported that in early diabetic neuropathy, features of axonal lesions, which exhibit a reduction in the amplitude, were observed first. Further, as the neuropathy progressed, a reduction in the amplitude and a decrease in the nerve conduction veloc- 
ity, both of which are due to demyelination and axonal injury, were observed. Kato et al. [20] performed a similar experiment in diabetes-induced rats for 4 weeks and a reduction in the sensory nerve conduction velocity was observed, but, histopathological changes were not found. Singhal et al. [21] found no pathological change during their 8-week experiment. Park et al. [11] performed a NCS in 60 diabetic patients and they reported that not only the conduction velocity but also the amplitude of compound motor action potential had decreased significantly.

No significant difference was found in the delay in latency and the reduction in the amplitude between 6 weeks and 7 weeks of preliminary experimentation. Thus, the delayed exercise group started exercise from the 7 th week in our study. A 14-week exercise program was conducted in which the parameters of nerve conduction studies had reached a plateau in 13 weeks. We made the decision to stop the NCS and dissected the tails of the rats for histopathologic examination at the 14th week.

Considering the relationship between the findings of the NCS and pathological findings in axonal degeneration, the amplitude decreases significantly/greatly, but the latency does not change much. In segmental demyelination, a delay in latency or a reduction in the nerve conduction velocity is more prominent than a reduction in the amplitude. In our study, the change in latency appeared before the change in the amplitude. Therefore, it can be assumed that the demyelination process occurs earlier than axonal degeneration. In addition, the delay in latency was in agreement with the histological findings, in which a reduction in the diameter of the myelin sheath was statistically more significant than a reduction in the axonal diameter.

Consequently, early induction of exercise could postpone the demyelination process in a nerve fiber in diabetic rats by reducing the complications such as hyperglycemia, dyslipidemia and inflammatory effects of obesity.

An early regular exercise program in addition to conventional insulin treatment was able to retard the progression of diabetic peripheral neuropathy.

In the future, prospective studies with larger sample sizes and long-term follow-ups are required to determine the irreversible changes in electrophysiological and histopathological findings.

\section{CONFLICT OF INTEREST}

No potential conflict of interest relevant to this article was reported.

\section{ACKNOWLEDGMENTS}

This study was supported by a grant from Inha University.

\section{REFERENCES}

1. Pyun SB, Kwon HK, Uhm CS. Effect of exercise on reinnervating soleus muscle after sciatic nerve injury in rats. J Korean Acad Rehabil Med 1999;23:1063-75.

2. Dumitru D. Electrodiagnostic medicine. 1st ed. Philadelphia: Mosby; 1995, p. 821-4.

3. Dyck PJ, Thomas PK. Diabetic neuropathy. 2nd ed. Philadelphia: W.B. Saunders; 1999. p. 222-36, 330-40.

4. Singleton JR, Smith AG, Marcus RL. Exercise as therapy for diabetic and prediabetic neuropathy. Curr Diab Rep 2015;15:120.

5. Malysz T, Ilha J, Nascimento PS, De Angelis K, Schaan BD, Achaval M. Beneficial effects of treadmill training in experimental diabetic nerve regeneration. Clinics (Sao Paulo) 2010;65:1329-37.

6. van Meeteren NL, Brakkee JH, Helders PJ, Wiegant VM, Gispen WH. Functional recovery from sciatic nerve crush lesion in the rat correlates with individual differences in responses to chronic intermittent stress. J Neurosci Res 1997;48:524-32.

7. Kwon HK, Lee HJ, Yim SK, Lee SR. Electrophysiologic assessement of axonopathy and demyelination in diabetic neuropathy according to the severity. J Korean Acad Rehabil Med 2002;26:50-4.

8. Kim MO, Kim SJ, Choi HC, Roh GH, Kim SY. Histochemical findings of soleus in relation to the severity of injury and duration of exercise in sciatic nerve injured rats. J Korean Acad Rehabil Med 2003;27:727-34.

9. Abu-Shakra SR, Cornblath DR, Avila OL, Chaudhry V, Freimer M, Glass JD, et al. Conduction block in diabetic neuropathy. Muscle Nerve 1991;14:858-62.

10. Said G. Diabetic neuropathy: a review. Nat Clin Pract Neurol 2007;3:331-40.

11. Park DW, Nam KS, Kim SC, Park SI, Choi E, Lee YG. Significance of amplitude and area ratio of compound 
muscle action potential in diagnosis of diabetic neuropathy. J Korean Acad Rehabil Med 2001;25:615-20.

12. Park BK, Kim SJ. Response of peripheral nerve to transient ischemia in streptozotocin-induced diabetic rats. J Korean Acad Rehabil Med 1993;17:392-405.

13. Kim CH, Choi HC, Roh GH. The preventive effect of nimodipine on the cisplatin induced neuropathy. J Korean Acad Rehabil Med 2003;27:90-5.

14. Gulsen I, Demiroglu M, Aycan A, Ucler R, Alaca I, Orhon ZN, et al. Effects of low-intensity treadmill exercise on sciatic nerve in experimental diabetic neuropathy. Anal Quant Cytopathol Histpathol 2016;38:95-102.

15. Kim WS, Lee SU. Harmful effect of land-based endurance exercise in rats with diabetic nerve. Med Sci Sports Exerc 2010;42:1625-31.

16. Kim MO, Yoon JS, Kwak JR, Choi HC, Roh GH, Kim SJ. Effect of therapeutic exercise according to degree of injury in sciatic nerve damaged rat. J Korean Acad Rehabil Med 2001;25:466-73.
17. Dyck PJ, Davies JL, Wilson DM, Service FJ, Melton LJ 3rd, O'Brien PC. Risk factors for severity of diabetic polyneuropathy: intensive longitudinal assessment of the Rochester Diabetic Neuropathy Study cohort. Diabetes Care 1999;22:1479-86.

18. Eugene B, Abthony SF, Dennis LK, Stephen LH, Dan LL. Harrison's principles of internal medicine. 15th ed. McGraw-Hill; 2001. p. 2109-37.

19. Hotta N, Koh N, Sakakibara F, Nakamura J, Hamara Y, Hara T, et al. Effects of propionyl-L-carnitine and insulin on the electroretinogram, nerve conduction and nerve blood flow in rats with streptozotocin-induced diabetes. Pflugers Arch 1996;431:564-70.

20. Kato N, Makino M, Mizuno K, Suzuki T, Shindo M. Serial changes of sensory nerve conduction velocity and minimal F-wave latency in streptozotocin-induced diabetic rats. Neurosci Lett 1998;244:169-72.

21. Singhal A, Cheng C, Sun H, Zochodne DW. Near nerve local insulin prevents conduction slowing in experimental diabetes. Brain Res 1997;763:209-14. 